\title{
Raman spectroscopy as an effective screening method for detecting adulteration of milk with small nitrogen-rich molecules and sucrose
}

\author{
M. K. Nieuwoudt, ${ }^{\star} \dagger \ddagger^{1}$ S. E. Holroyd,§ C. M. McGoverin,\#ll M. C. Simpson, ${ }^{*} \dagger \ddagger \# l l$ and D. E. Williams ${ }^{*} \ddagger$ \\ *MacDiarmid Institute for Advanced Materials and Nanotechnology, New Zealand \\ †The Photon Factory, and \\ $\ddagger$ School of Chemical Sciences, The University of Auckland, 23 Symonds St., Auckland, 1142 New Zealand \\ §Fonterra Research \& Development Centre, Private Bag 11029, Palmerston North, New Zealand \\ \#The Dodd Walls Centre for Photonic and Quantum Technologies, New Zealand \\ IIDepartment of Physics, The University of Auckland, 23 Symonds St., Auckland, 1142 New Zealand
}

\begin{abstract}
Adulteration of milk for commercial gain is acknowledged as a serious issue facing the dairy industry. Several analytical techniques can be used to detect adulteration but they often require time-consuming sample preparation, expensive laboratory equipment, and highly skilled personnel. Here we show that Raman spectroscopy provides a simple, selective, and sensitive method for screening milk, specifically for small nitrogen-rich compounds, such as melamine, urea, ammonium sulfate, dicyandiamide, and for sucrose. Univariate and multivariate statistical methods were used to determine limits of detection and quantification from Raman spectra of milk spiked with 50 to $1,000 \mathrm{mg} / \mathrm{L}$ of the N-rich compounds and 0.25 to $4 \%$ sucrose. Partial least squares (PLS) calibration provided limit of detection minimum thresholds $<200 \mathrm{mg} / \mathrm{L}(0.02 \%)$ for the 4 N-rich compounds and $<0.8 \%$ for sucrose, without the need for surface-enhanced Raman spectroscopy. The results show high reproducibility (7\% residual standard deviation) and $100 \%$ efficiency for screening of milk for these adulterants.
\end{abstract}

Key words: milk adulteration, Raman spectroscopy, nitrogen-rich molecules, sucrose

\section{INTRODUCTION}

The adulteration of milk for economic gain has been an ongoing problem for more than 150 yr: dilution of milk with water was reported as early as 1857 (Gem, 1857) and, in 1890, The New York Times exposed the addition of compounds such as borax, soda, or salicylic acid to increase milk shelf life (New York Times, 1890). Unfortunately, adulteration of milk is still prevalent today with the fraudulent addition of a variety of

Received September 1, 2015.

Accepted December 10, 2015.

${ }^{1}$ Corresponding author: m.nieuwoudt@auckland.ac.nz chemicals (Moore et al., 2012; Cattaneo and Holroyd, 2013; Santos et al., 2013). Recently, small nitrogen-rich compounds such as melamine (1,2,4-triazine) have been fraudulently added to milk to artificially boost its apparent protein content (Chan et al., 2008), which is routinely measured as total nitrogen content using the Kjeldahl or Dumas analytical test (Codex Alimentarius, 2010; Moore et al., 2010). As these test methods cannot distinguish between nitrogen in milk protein and other nitrogen compounds, more sophisticated laboratory test methods are necessary for sensitive detection of these compounds in milk. These include GC, HPLC, and hydrophilic interaction liquid chromatography (HILIC) methods, with MS detection (Desmarchelier et al., 2009; Abernethy and Higgs, 2013; Domingo et al., 2014). These techniques can measure melamine concentrations well below the World Health Organization's maximum residue levels of $2.5 \mathrm{mg} / \mathrm{L}$ in milk and $1.0 \mathrm{mg} / \mathrm{L}$ in infant formula (WHO, 2009). However, they require lengthy pretreatment of the milk samples, including centrifugation and addition of chemicals to remove the protein fraction, expensive laboratory equipment, and highly skilled operators.

Spectroscopic methods that do not require such sample preparation steps have been investigated as rapid and "greener" alternative methods for detecting these compounds in liquid or powdered milk; these include mid- and near-infrared spectroscopy (Mauer et al., 2009; Balabin and Smirnov, 2011; Cattaneo and Holroyd, 2013; Jawaid et al., 2013), Raman spectroscopy (Okazaki et al., 2009; Cheng et al., 2010), hyperspectral Raman imaging (Qin et al., 2010, 2011, 2012, 2013a,b; Kim et al., 2013), and Fourier transform-Raman spectroscopy (Srilakshmi et al., 2007; Cheng et al., 2010, 2012; Chao et al., 2013). Mid- and near-infrared spectroscopy combined with chemometric analysis have already been shown to be effective as a rapid, sensitive, robust, and low-cost alternative for analysis of melamine in milk and infant formula (Balabin and Smirnov, 2011; Abbas et al., 2013; Jawaid et al., 2013; 
Santos et al., 2013). However, different studies show considerable variation in the root mean square error of calibration and limit of detection (LOD) due to variations in instrumentation and method used for the measurements.

Compared with infrared spectra, Raman spectra generally contain fewer, sharper, and more-discrete bands that are significantly stronger than those from water. This affords Raman spectroscopy several advantages over infrared absorption: it enables greater sensitivity in measurement of low concentrations and the discrete and sharper spectral bands make Raman spectroscopy far more specific for the simultaneous analysis of mixtures of different adulterants in milk. Another advantage is the variety of robust mini and portable Raman systems that have become available commercially at increasingly competitive prices. For example, a portable mini Raman system with microscope and surface-enhanced Raman spectroscopy (SERS) has already been successfully used to detect melamine as a contaminant in eggs (Cheng and Dong, 2011). Combining partial least squares (PLS) and partial least squares discriminant analysis (PLS-DA) multivariate analysis methods with such mini Raman systems shows promise for the application of Raman spectroscopy as a robust technique for qualitative and quantitative screening of adulterants in milk. Further, the suitability of Raman microscopy with micro-fiber optics for analyzing aqueous fluids in microfluidics extends the usefulness of application to on-site analysis of milk, as has already been described for biological and medical screening applications (Johansson et al., 2007; Ashok et al., 2010; Chrimes et al., 2013).

The disadvantage of Raman spectroscopy for liquid milk is the relatively low signal-to-noise ratio (SNR) due to diffuse scattering by the milk matrix. Raman analysis has therefore been limited to powdered or dried milk. However, in these media, LOD are poor: those measured for melamine, dicyandiamide (DCD), ammonium sulfate (AmS), and urea in milk are between 1,000 and 1,300 mg/L (Cheng et al., 2010; Qin et al., 2013a,b). More sensitive detection of melamine in milk has been achieved using SERS, which utilizes the enhanced electromagnetic fields and chemical enhancement provided by gold or silver nanostructures; LOD as low as $2.4 \mu \mathrm{g} / \mathrm{L}$ have been reported (Zhao et al., 2013). However, most of the SERS measurements in the literature require sample preparation steps similar to those for HPLC, such as centrifugation and addition of chemicals to remove the protein fraction that may block analyte binding sites on SERS substrates. An exception is a SERS study using simple dilution of milk to which silver nanoparticles were added: a melamine LOD of $1 \mathrm{mg} / \mathrm{L}$ was obtained (Rajapandiyan et al.,
2015). Another disadvantage of SERS is low reproducibility (Guicheteau et al., 2011), which is also highly variable, due to the stochastic nature of the "hotspots" of intense electromagnetic radiation between the nanostructures (Moskovits, 2013). Variations can be as high as $\pm 40 \%$ (Norrod et al., 1997).

The aim of this study was to find a robust, simple, and reliable method using Raman spectroscopy to screen for the adulteration of milk with N-rich compounds, without the need for SERS, dilution, or other preparation steps. Although baseline levels of these compounds in foods require more sensitive detection such as laboratory chromatographic techniques or SERS, adulteration levels used for commercial gain are hypothesized to be much higher, at levels between 90 and 4,000 mg/L (Abernethy and Higgs, 2013). The focus of the study was to improve both the reproducibility and sensitivity of Raman analysis for 4 N-rich compounds (melamine, DCD, AmS, and urea) and sucrose in milk without the use of SERS. Collection optics, instrument parameters, and sampling methods were optimized to improve reproducibility and sensitivity. Sucrose is often added to increase the solids content and sweeten milk, and is currently analyzed using HPLC with refractive index detection (Chávez-Servín et al., 2004) or using a modified Sewilanoff method requiring addition of harmful chemicals (Food Safety and Standard Authority of India, 2012). As far as we are aware, no quantitative study using Raman analysis of sucrose in milk has yet been reported.

\section{MATERIALS AND METHODS}

\section{Sample Preparation}

A commercial brand of homogenized full-cream milk was used for all experiments. Adulterated milk solutions were prepared by dissolving the appropriate amount of powdered adulterant in $100 \mathrm{~mL}$ of milk. The concentration of sucrose was varied between 0 and $40,000 \mathrm{mg} / \mathrm{L}$ to give a total of 7 samples, and 3 spectra were recorded for each sample, resulting in 21 spectra that were used for both univariate analysis and PLS analysis. For the 4 small N-rich molecules (melamine, $\mathrm{AmS}, \mathrm{DCD}$, and urea), the concentration of each was varied between 0 and $1,000 \mathrm{mg} / \mathrm{L}$ to give a total of 60 samples. Of these, 30 samples containing only one adulterant each and were used for univariate analysis, whereas the 30 samples that included additional mixtures were used for the PLS and PLS-DA models, 12 of which were pure milk samples from different dates over a period of 10 mo. Spectra were recorded from 2 different aliquots from each sample, resulting in 120 spectra in total. For the PLS and PLS-DA calibrations, these 
were divided into a training set of 80 spectra and a separate validation set of 40 spectra, by selecting every third sample (row) in the data matrix for the validation set. An additional 4 spectra recorded of sucrose in milk $(2,500$ to $20,000 \mathrm{mg} / \mathrm{L})$ were included in the validation set for the PLS-DA.

To assess the partitioning of the investigated N-rich small molecules into the cream fraction, $3,000 \mathrm{mg} / \mathrm{L}$ solutions of melamine (BDH Chemicals Ltd., Poole, UK), urea, and AmS (ECP Ltd., Auckland, New Zealand) and a $5,000 \mathrm{mg} / \mathrm{L}$ solution of DCD (Sigma-Aldrich Co., St Louis, MO) were made. Two milliliters of each solution was centrifuged at $8,050 \times g$ for $15 \mathrm{~min}$, and spectra were recorded from the cream fraction.

\section{Raman Spectroscopy}

Drops of each sample $(15 \mu \mathrm{L})$ were placed individually into 4-mm chrome mirror-based sampling wells of a Thermo (Thermo Fisher Scientific, Waltham, MA) sample slide. These wells constrained the drops into 4-mm-diameter circles that dried over $1 \mathrm{~h}$ to an average thickness of $112 \mu \mathrm{m}$. Raman spectra were recorded of the dried drops in backscattered configuration using a Renishaw System 1000 Raman microprobe (Renishaw plc, Wotton-under-Edge, UK) with a $25-\mathrm{mW}$ $785-\mathrm{nm}$ laser and $50 \times$ objective, to give a spot size of $2 \mu \mathrm{m}$ at the center and $2.5 \mathrm{~mW}$ at the sample. The use of this excitation wavelength avoided fluorescence by milk proteins that would obscure the Raman bands. Reproducibility of the signal was optimized by keeping the sampling volume constant and recording spectra from the center of each dried drop. Reproducibility was parameterized as the relative standard deviation (\%RSD) calculated from 3 spectra recorded from different drops of the same solution. The average thickness of the milk drops of $112 \mu \mathrm{m}$ was significantly greater than the depth of focus, $\Delta f$, of the focused laser spot, which was determined to be $8 \mu \mathrm{m}$ (Delhaye et al., 1996):

$$
\Delta f=\frac{\pi(d)^{2}}{2 \lambda}
$$

where $d$ is the laser spot diameter and $\lambda$ is the laser excitation wavelength.

For the backscattered geometry of the Raman microprobe, the intensity of the Raman spectra depends on several factors: laser power density, sample properties and geometry, spectrometer collection parameters, and integration time (McCreery, 2000). In our system, the only parameters that could be varied were the sample volume, collection optics, and laser power, limited to a maximum of $25 \mathrm{~mW}$. Under this constraint, a minimum integration time of $240 \mathrm{~s}$ was required for an adequate SNR of 3 for $200 \mathrm{mg} / \mathrm{L}$ of melamine. The optimum entrance slit width with acceptable resolving power for the Raman urea band at $1,008 \mathrm{~cm}^{-1}$ and milk protein phenylalanine $1,001 \mathrm{~cm}^{-1}$ band was $75 \mu \mathrm{m}$. The spectral range obtained with $1,200 \mathrm{~mm}^{-1}$ grating in static mode and $785 \mathrm{~nm}$ excitation was $670 \mathrm{~cm}^{-1}$. Due to overlap of some of the bands of melamine, DCD, urea, AmS, and sucrose with the milk bands, an averaged spectrum of 3 pure milk samples was subtracted from each adulterated milk spectrum to enable more accurate measurement of peak height for the univariate analysis. Peak heights were measured from these difference spectra at $683 \mathrm{~cm}^{-1}$ for melamine, $976 \mathrm{~cm}^{-1}$ for AmS, 1,008 $\mathrm{cm}^{-1}$ for urea, $836 \mathrm{~cm}^{-1}$ for sucrose, and $2,198 \mathrm{~cm}^{-1}$ for DCD. The latter band was used for univariate analysis of DCD as the band at $926 \mathrm{~cm}^{-1}$ overlapped with the milk bands (Figure 1). For PLS and PLS-DA, only the region 1,100 to $550 \mathrm{~cm}^{-1}$ was used.

\section{PLS and PLS-DA}

Partial least squares calibrations are generally more accurate and robust than univariate calibrations. These multivariate calibrations avoid the inaccuracies introduced in univariate analyses when the band due to the analyte of interest overlaps with bands of other analytes or milk, primarily because information from the entire spectrum is considered instead of only a single peak height. The PLS factors are calculated to maximize covariance between the spectral and analyte concentration matrices thereby excluding nonanalyte signal contributions such as instrument noise, consequently making the calibration more robust than univariate methods.

Spectral data were preprocessed with a 3-point baseline correction using the Omnic 7.1 spectroscopic software (Thermo Fisher Scientific) and smoothing using a Savitsky-Golay second-derivative algorithm (secondorder polynomial over 9 points) in the Unscrambler X software (Camo Software AS, Oslo, Norway). After preprocessing, the data set was divided into a training set consisting of 80 spectra and a validation set of 40 spectra, by selecting every third sample (row) in the data matrix. Partial least squares 1 (PLS1) calibrations for melamine, AmS, DCD, and urea were calculated using the spectral region 600 to $1,092 \mathrm{~cm}^{-1}$. Root mean square error of cross validation (RMSECV) was determined using an internal leave-one-out cross validation. These calibration models were then used to predict the concentrations of the separate validation 
set. Each PLS1 calibration model was evaluated from the RMSECV for internal validation and the standard error of prediction (SEP) and ratio of prediction to standard deviation (RPD) for the separate validation prediction. The RPD is a nondimensional statistic used to evaluate PLS calibration models (Williams, 2008) and is calculated by dividing the standard deviation of the reference values used in the validation by the SEP. Values for RPD between 3.1 and 4.9 classify the model as suitable for screening, whereas values between 5.0 and 6.4 are suitable for quality control. Values between 6.5 and 8.0 classify a model as suitable for process control, and values of 8.1 or greater are considered suitable for any application. A separate PLS calibration model for sucrose was created from the training set of 21 spectra recorded from 3 separate drops of each of 7 different concentrations between 0 and 40,000 mg/L. For sucrose, the RMSECV for the internal leave-one-out cross validation was used instead of a separate validation set.

The PLS-DA calibration model was created using the same training set as for PLS calibrations; however, the validation set included an additional 4 sucrose spectra.
For the PLS-DA training set, a dummy concentration matrix was drawn up with values of -1 assigned to the pure milk spectra and +1 to the milk solutions containing one or more of melamine, AmS, DCD, and urea.

\section{Limits of Detection and Quantification}

The LOD and limit of quantification (LOQ) of the univariate analyses were measured according to International Conference on Harmonisation guidelines (ICH, 1996): $\mathrm{LOD}=(3.3 \sigma) / S$ and $\mathrm{LOQ}=(10 \sigma) / S$, where $\sigma$ $=$ standard deviation $(\mathbf{S D})$ of the response and $S=$ slope of the calibration curve of peak height vs. concentration; $\sigma$ can be the SD of the blank (measured from the baseline noise for a blank over the range of the peak of interest) or the SD of the regression line, or the SD of the $y$-intercepts of the regression line. In this study, $\sigma$ was the SD of the regression line as the LOD and LOQ values obtained compared best with visual estimation of the spectra and were most conservative.

For multivariate methods such as PLS calibration, the LOD can be roughly estimated as $3 \times$ root mean SEP (Cattaneo and Holroyd, 2013). A more rigorous

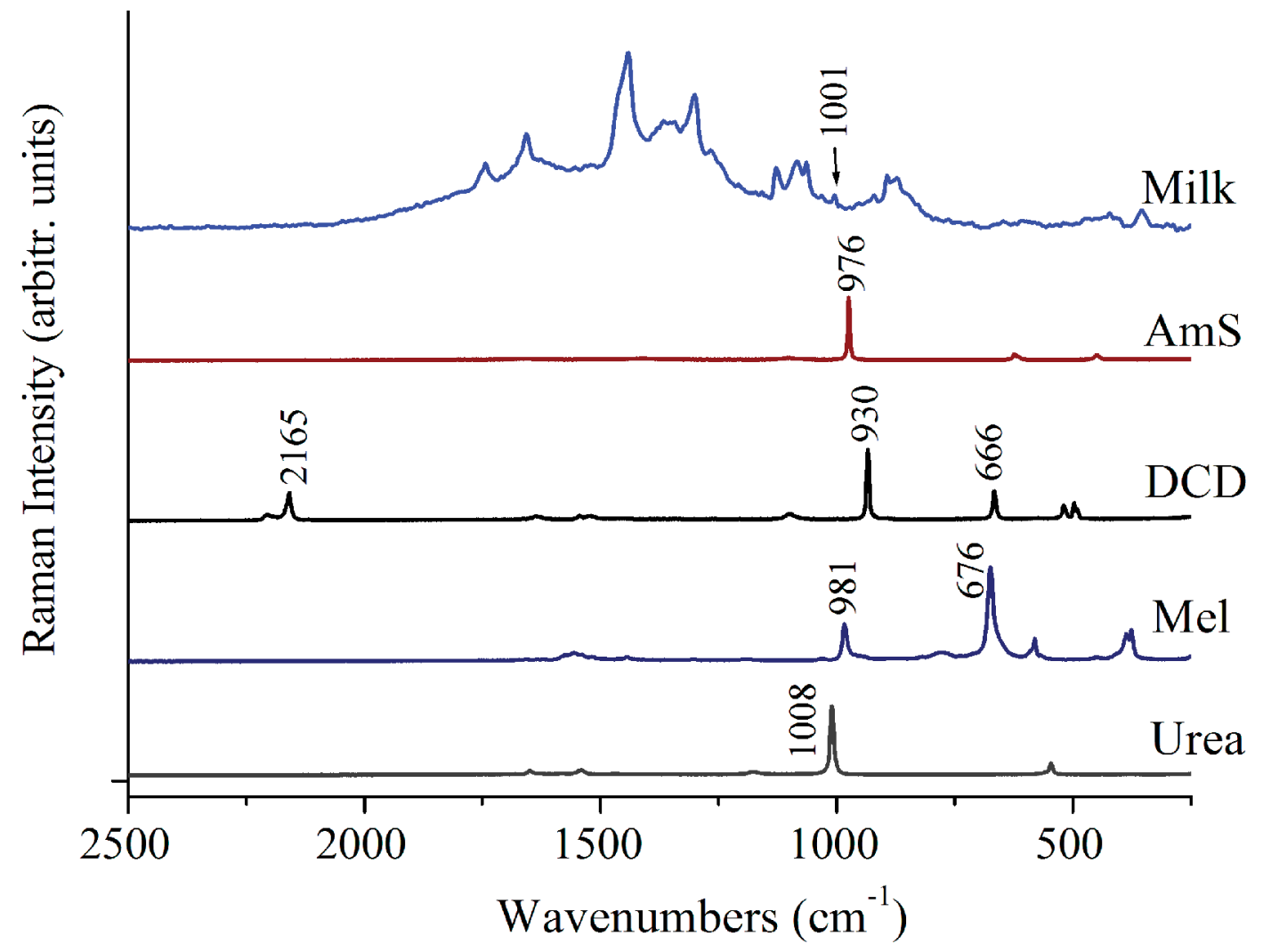

Figure 1. Spectra of the 4 N-rich adulterants: melamine (Mel), ammonium sulfate (AmS), dicyandiamide (DCD), and urea, and milk in the region 2,500 to $250 \mathrm{~cm}^{-1}$. The strongest bands of each of the 4 N-rich compounds are labeled, as well as the $1,001 \mathrm{~cm}^{-1}$ phenylalanine band of milk. The spectra have been offset for clarity. Color version available online. 
and IUPAC (International Union of Pure and Applied Chemistry)-consistent approach defines a set of LOD that are based on the set of samples in the training set and characteristic for the overall PLS calibration model (Allegrini and Olivieri, 2014). The LOD interval is obtained by finding the limits of the region in the hyperplane, $\pi_{0}$, representing samples with zero analyte concentration, or sample blanks. This region is a function of the variance in the predicted analyte concentration for a blank sample, which in turn is a function of $h_{0}$, the distance between $\pi_{0}$ and the center of a normalized calibration score space. Although these sample blanks contain none of the analyte of interest, they may well contain other analytes, and the LOD interval is determined by finding the smallest and largest extrapolated leverages to zero analyte concentration, $h_{0 \min }$ and $h_{0 \max }$, for each calibration set. These values are then used to determine $\mathrm{LOD}_{\min }$ and $\mathrm{LOD}_{\max }$, from (Allegrini and Olivieri, 2014):

$$
\begin{gathered}
\mathrm{LOD}_{\text {min }}=3.3\left[\mathrm{SEN}^{-2} \operatorname{var}(x)+h_{0 \text { min }} \mathrm{SEN}^{-2} \operatorname{var}(x)\right. \\
\left.+h_{0 \text { min }} \operatorname{var}\left(y_{\text {cal }}\right)\right]^{1 / 2} \\
\mathrm{LOD}_{\text {max }}=3.3\left[\mathrm{SEN}^{-2} \operatorname{var}(x)+h_{0 \text { max }} \mathrm{SEN}^{-2} \operatorname{var}(x)\right. \\
\left.+h_{0 \text { max }} \operatorname{var}\left(y_{\text {cal }}\right)\right]^{1 / 2}
\end{gathered}
$$

where SEN is the inverse of the Euclidean norm of the regression coefficient, $\operatorname{var}\left(y_{c a l}\right)$ is the variance in calibration concentrations, obtained here from the crossvalidation variance, $\operatorname{var}(x)$ is the variance in instrumental signals, which is the square of the signal noise, measured for a region of the baseline in the mean blank spectrum with no peaks, and $h_{0 \min }$ and $h_{0 \max }$ are the maximum and minimum extrapolated to zero leverages of each calibration training set.

Moreover, $h_{0 \min }$ can be measured as follows:

$$
h_{0 \min }=\frac{\bar{y}_{c a l}^{2}}{\sum_{i=1}^{l} y_{i}^{2}},
$$

where $y_{i}$ is the mean centered concentration for the $i$ th calibration sample; and $h_{0 \max }$ is the maximum $h_{0 \text { cal }}$ value for the calibration set, obtained for each sample in the calibration set:

$$
h_{0 c a l}=h_{c a l}+h_{0 m i n} 1-\left(\frac{y_{c a l}}{\bar{y}_{c a l}}\right)^{2} .
$$

Similarly, the LOQ interval ( $\min$ and $\max$ ) can be determined as follows:

$$
\begin{gathered}
\mathrm{LOQ}_{\text {min }}=10\left[\mathrm{SEN}^{-2} \operatorname{var}(x)+h_{0 \text { min }} \mathrm{SEN}^{-2} \operatorname{var}(x)\right. \\
\left.+h_{0 \text { min }} \operatorname{var}\left(y_{\text {cal }}\right)\right]^{1 / 2} \\
\mathrm{LOQ}_{\max }=10\left[\mathrm{SEN}^{-2} \operatorname{var}(x)+h_{0 \text { max }} \mathrm{SEN}^{-2} \operatorname{var}(x)\right. \\
\left.+h_{0 \text { max }} \operatorname{var}\left(y_{\text {cal }}\right)\right]^{1 / 2} .
\end{gathered}
$$

These values can be used to evaluate whether the predicted concentration of an unknown sample is below or above the detection limit. For samples with predicted concentrations falling within the range $\mathrm{LOD}_{\text {min }}>y_{i}<$ $\mathrm{LOD}_{\max }$, an LOD for the specific sample can be evaluated individually by approximating its real leverage, $h$, to the leverage $h_{0}$, thus treating the sample as if it were a blank. The LOD obtained would then be used to check whether the analyte is present or absent depending on whether its predicted concentration is above or below the sample-specific LOD (Allegrini and Olivieri, 2014).

\section{RESULTS AND DISCUSSION}

Figure 1 shows the spectra of milk and pure samples of melamine, AmS, DCD, and urea. The strongest band for melamine appeared at $676 \mathrm{~cm}^{-1}$ and was due to in-plane deformation of the triazine ring, associated with $\mathrm{C}-\mathrm{N}$ stretching of the $\mathrm{C}-\mathrm{NH}_{3}$ groups. The weaker band at $986 \mathrm{~cm}^{-1}$ is the ring-breathing mode due to C-N stretching of the triazine ring (Koglin et al., 1996). For DCD, 2 bands appeared at 2,198 $\mathrm{cm}^{-1}$ and 2,165 $\mathrm{cm}^{-1}$ due to $\mathrm{C}=\mathrm{N}$ stretching, whereas another 2 bands occurred at $933 \mathrm{~cm}^{-1}$ and $668 \mathrm{~cm}^{-1}$ due to $\mathrm{N}-\mathrm{C}-\mathrm{N}$ stretching and $\mathrm{NH}_{2}$ deformation, respectively (Lin et al., 2015). The strongest band in the spectrum of urea occurs at $1,008 \mathrm{~cm}^{-1}$ and is due to the $\mathrm{N}-\mathrm{C}-\mathrm{N}$ symmetric stretch (Keuleers et al., 1999), whereas for AmS, the band at $976 \mathrm{~cm}^{-1}$ is due to the symmetric stretch mode of $\mathrm{SO}_{4}{ }^{2-}$, and the much weaker band at $620 \mathrm{~cm}^{-1}$ is due to the triply degenerate $\mathrm{SO}_{4}{ }^{2-}$ deformation mode (Kettle et al., 1984).

The $982 \mathrm{~cm}^{-1}$ melamine and $976 \mathrm{~cm}^{-1}$ AmS bands are close in frequency, as are the melamine $683 \mathrm{~cm}^{-1}$ and DCD $666 \mathrm{~cm}^{-1}$, and urea $1,008 \mathrm{~cm}^{-1}$ and milk protein phenylalanine $1,001 \mathrm{~cm}^{-1}$ bands. In mixtures of these compounds, the bands overlapped (Figure A1, Appendix), which introduced inaccuracies in peak height measurements from spectra of milk solutions adulterated with 2 or more of these compounds. This led to nonlinear calibration plots of peak height versus concentration, particularly for melamine and ammonium sulfate. Therefore, for reliable determination of LOD and LOQ from univariate measurement of peak height, spectra were recorded of milk samples spiked 
individually with each compound to minimize component overlap.

\section{Univariate Calibration Models}

Figure 2 shows the spectra of milk spiked separately with different concentrations (50 to $1,000 \mathrm{mg} / \mathrm{L}$ ) of melamine, AmS, DCD, and urea. Two different regions are shown for DCD, as strong bands in the $2,100 \mathrm{~cm}^{-1}$ region were isolated from any of the milk bands, which makes this region more suitable for univariate measurement of peak height. We note that some of the adulterant band frequencies and relative intensities shifted or changed when they were dissolved in milk; in particular, the melamine $676 \mathrm{~cm}^{-1}$ band shifted to $683 \mathrm{~cm}^{-1}$, the DCD $930 \mathrm{~cm}^{-1}$ band shifted to $926 \mathrm{~cm}^{-1}$, and the smaller 2,198 $\mathrm{cm}^{-1}$ DCD band increased to a greater intensity than that of the $2,165 \mathrm{~cm}^{-1}$ band.

Sucrose-specific peaks within milk were only observed for higher concentrations (2,500 to 40,000 mg/L). Because of the poorer Raman scattering cross-section of sucrose, its Raman bands were much weaker and broader than those of the 4 N-rich compounds; sucrose also exhibited a greater degree of overlap with the milk component bands (Figure 3a). An extended spectral range is shown for sucrose between 500 and $1,800 \mathrm{~cm}^{-1}$ to include all the strongest bands of sucrose, which can be more clearly seen in the difference spectra in Figure $3 \mathrm{~b}$ and in the spectrum of sucrose granules in Figure 3c. The $836 \mathrm{~cm}^{-1}$ band was used for peak height measurements as this band showed the least overlap with the milk component bands.

Calibration plots of peak height versus concentration were constructed for each of the 5 components and are shown in Figure A2 in the Appendix. The plots showed good linearity over the whole concentration range; the $\mathrm{R}^{2}$ values, \%RSD, LOD, and LOQ determined from the slopes are given in Table 1.

The LOD obtained for the each of the 4 N-rich compounds in milk are 4 to 5 times lower than those from Raman imaging experiments and Fourier transform Raman measurements of powdered milk samples (Cheng et al., 2010, 2012; Qin et al., 2010, 2012, 2013a,b; Chao et al., 2013). For sucrose, the LOD of $7,290 \mathrm{mg} / \mathrm{L}$ $(0.7 \%)$ is an improvement on the results from a nearinfrared study of sucrose in milk powder (Borin et al., 2006) for which the LOD can be approximated as $3 \times$ RMSECV and was 0.86 to $2.58 \%$. Good linearity was obtained for each compound over the whole concentration range investigated, and the LOD were close to the lower limits expected for adulterated milk, especially in the case of DCD and melamine. The improvement in LOD was due to improving the SNR by optimizing the slit width, sample presentation, using a high numerical aperture microscope objective, and a 4-min integration time. The reproducibility afforded by the consistent sampling volume of $15 \mu \mathrm{L}$ resulted in a \%RSD average of $7 \%$ for the 4 N-rich compounds and $3 \%$ for sucrose, lower than the reproducibilities previously reported for SERS (Norrod et al., 1997; Guicheteau et al., 2011). The $15-\mu \mathrm{L}$ sample volume allowed reproducible filling of the wells and although we did not explore smaller volumes, they would not be expected to affect signal intensity unless the drop dried to a thickness close to or less than the depth of focus $(8 \mu \mathrm{m})$. The criticality of the volumes and fragility of the sampling method could be explored in a future study by experimenting with volume sizes close to this to determine the effect on signal intensity. The only 3 limitations to a smaller drop size would be that (1) the drop needs to fill the well reproducibly and evenly, which may not happen spontaneously with smaller volumes, (2) the drop must dry evenly to a reproducibly flat surface, and (3) the thickness of the dried drop should not be less than the depth of focus of the laser.

Calibration plots were used to determine the fraction of melamine, DCD, AmS, and urea in the cream fraction after centrifugation of milk solutions containing 3,000 $\mathrm{mg} / \mathrm{L}$ of one of either melamine, AmS, or urea, and $5,000 \mathrm{mg} / \mathrm{L}$ of DCD. Spectra were recorded of these solutions in the region from 1,150 to $560 \mathrm{~cm}^{-1}$ before and after centrifugation (Figure A3, Appendix). For DCD, the spectra were recorded in the 2,300 to $1,980 \mathrm{~cm}^{-1}$ region. The percentages of each compound remaining in the cream fraction after centrifugation were determined from peak heights in difference spectra, using the calibration plots and are given in Table 2 .

The results show that a significant proportion of the compound remains in the cream fraction after centrifuging, particularly for melamine and DCD. The greater solubility of these 2 compounds in the cream fraction was expected as they are more nonpolar than urea and ammonium sulfate, with the latter being the most polar and thus least fat soluble, which explains its relatively low concentration in the fat fraction. The results show the need to consider the possibility of introducing inaccuracies in measurement of milk solutions using techniques that require centrifugation in the preparation steps.

\section{PLS Calibrations}

The 120 spectra used in the training and validation sets for the PLS analysis of the 4 N-rich compounds and for the PLS-DA, with an additional 4 sucrose validation spectra, are plotted in Figure 4. The spectra have been baseline-corrected and smoothed, and the strongest bands for each component have been labeled. 
NIEUWOUDT ET AL.
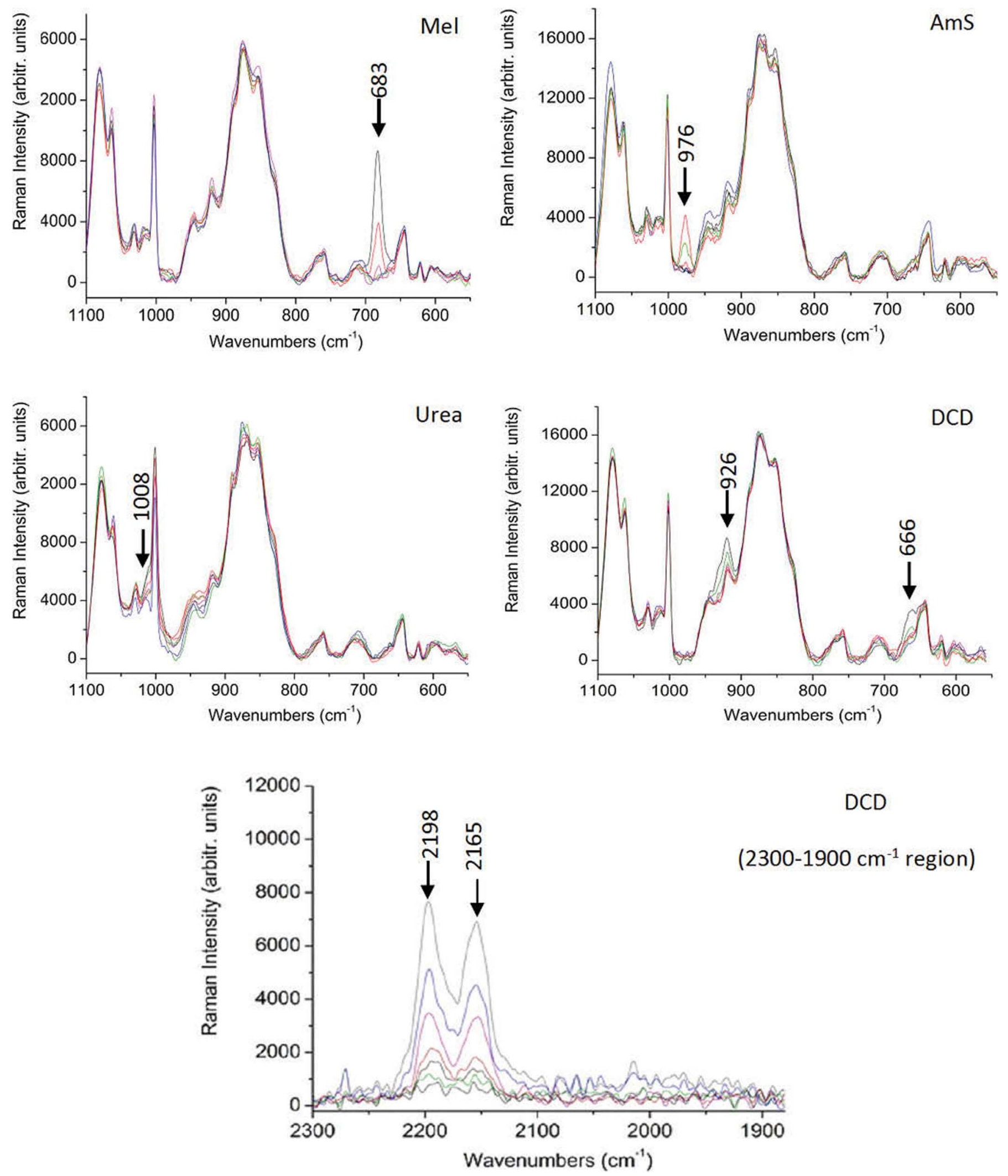

Figure 2. The spectra of milk solutions spiked with single components melamine (Mel), ammonium sulfate (AmS), dicyandiamide (DCD), and urea at concentrations between 0 and $1,000 \mathrm{mg} / \mathrm{L}$. The bottom spectrum shows DCD-spiked milk in the region 2,300 to $1,900 \mathrm{~cm}^{-1}$. The peak positions of the strongest adulterant bands are labeled in each spectrum. Color version available online. 
Table 1. Limit of detection (LOD), limit of quantification (LOQ), coefficient of determination $\left(\mathrm{R}^{2}\right)$, and residual standard deviation (RSD) from calibration plots of peak height versus concentration

\begin{tabular}{lccccc}
\hline Item & Melamine & $\mathrm{AmS}^{1}$ & $\begin{array}{c}\mathrm{DCD}^{2} \\
\left(2,198 \mathrm{~cm}^{-1}\right)\end{array}$ & Urea & Sucrose \\
\hline LOD $(\mathrm{mg} / \mathrm{L})$ & 180 & 230 & 130 & 280 & $7,290(0.7 \%, \mathrm{wt} / \mathrm{wt})$ \\
LOQ $(\mathrm{mg} / \mathrm{L})$ & 560 & 710 & 410 & 840 & $22,090(2.2 \%, \mathrm{wt} / \mathrm{wt})$ \\
$\mathrm{R}^{2}$ & 0.98 & 0.99 & 0.99 & 0.97 & 0.98 \\
RSD $(\%)$ & 5.0 & 7.9 & 10.6 & 7.9 & 3.4 \\
\hline
\end{tabular}

${ }^{1}$ Ammonium sulfate.

${ }^{2}$ Dicyandiamide.

The figure clearly shows the extent of overlap of the strongest bands for the 5 different components with each other and with those of the milk. The PLS1 calibration regression coefficients for the optimum number of factors determined for each compound are plotted in Figure 5. As expected, the largest regression coefficients for each model were coincident with the spectral region of greatest intensity for the analyte of interest.
For melamine and AmS, 3 PLS factors were used for the calibration; these accounted for 97 and $93 \%$ of the variance in the data, respectively. In contrast, DCD and urea required more factors, with 5 PC proving optimum for DCD, explaining $97 \%$ of the data variance, and $6 \mathrm{PC}$ for urea, explaining $92 \%$ of the data variance.

The PLS calibration model predicted concentrations versus reference concentrations for the validation set

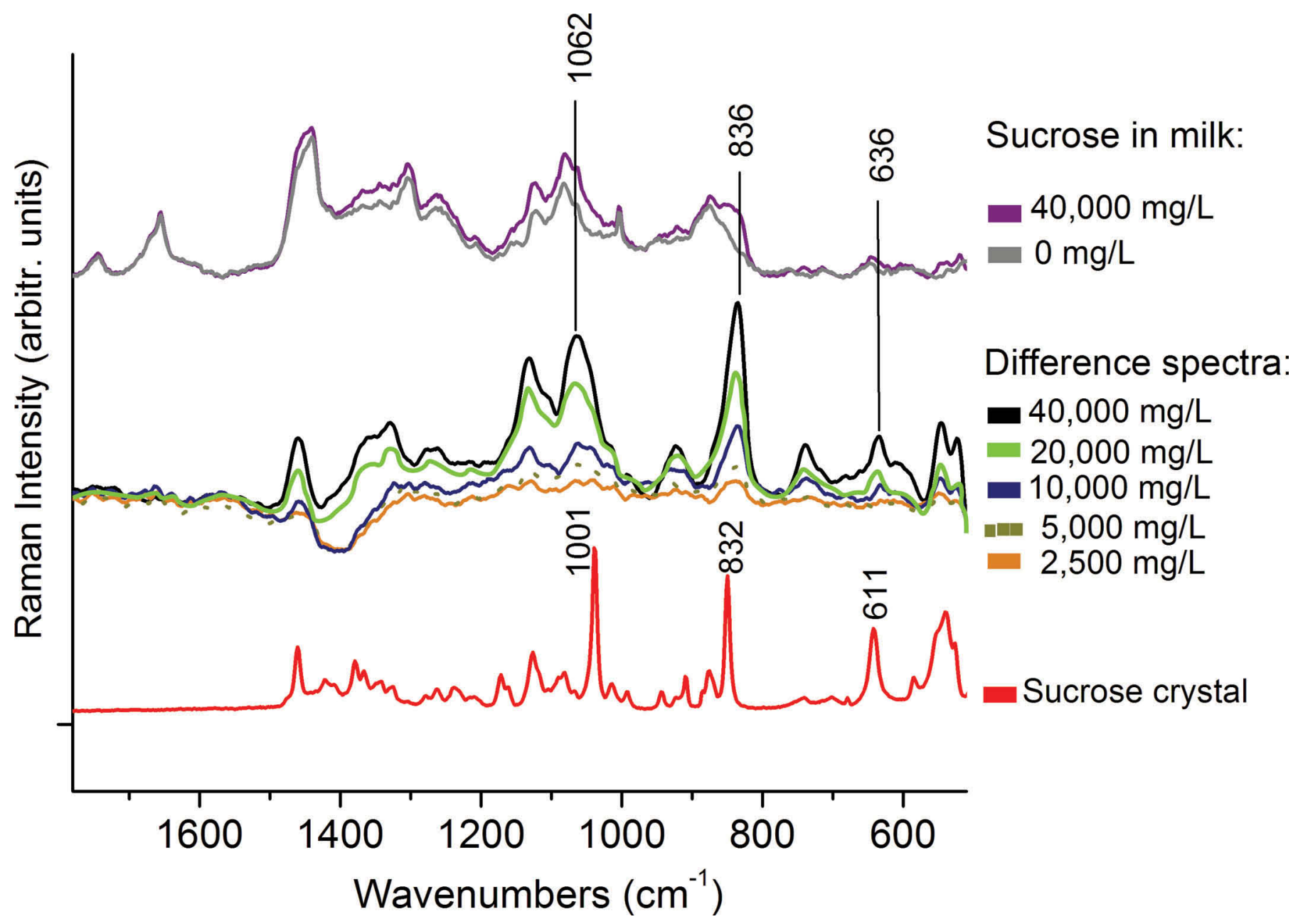

Figure 3. (a) Spectra of milk spiked with 40,000 and $0 \mathrm{mg} / \mathrm{L}$ of sucrose, (b) difference spectra of 5 solutions ranging from 2,500 to 40,000 $\mathrm{mg} / \mathrm{L}$, and (c) spectrum of a sucrose crystal. Color version available online. 


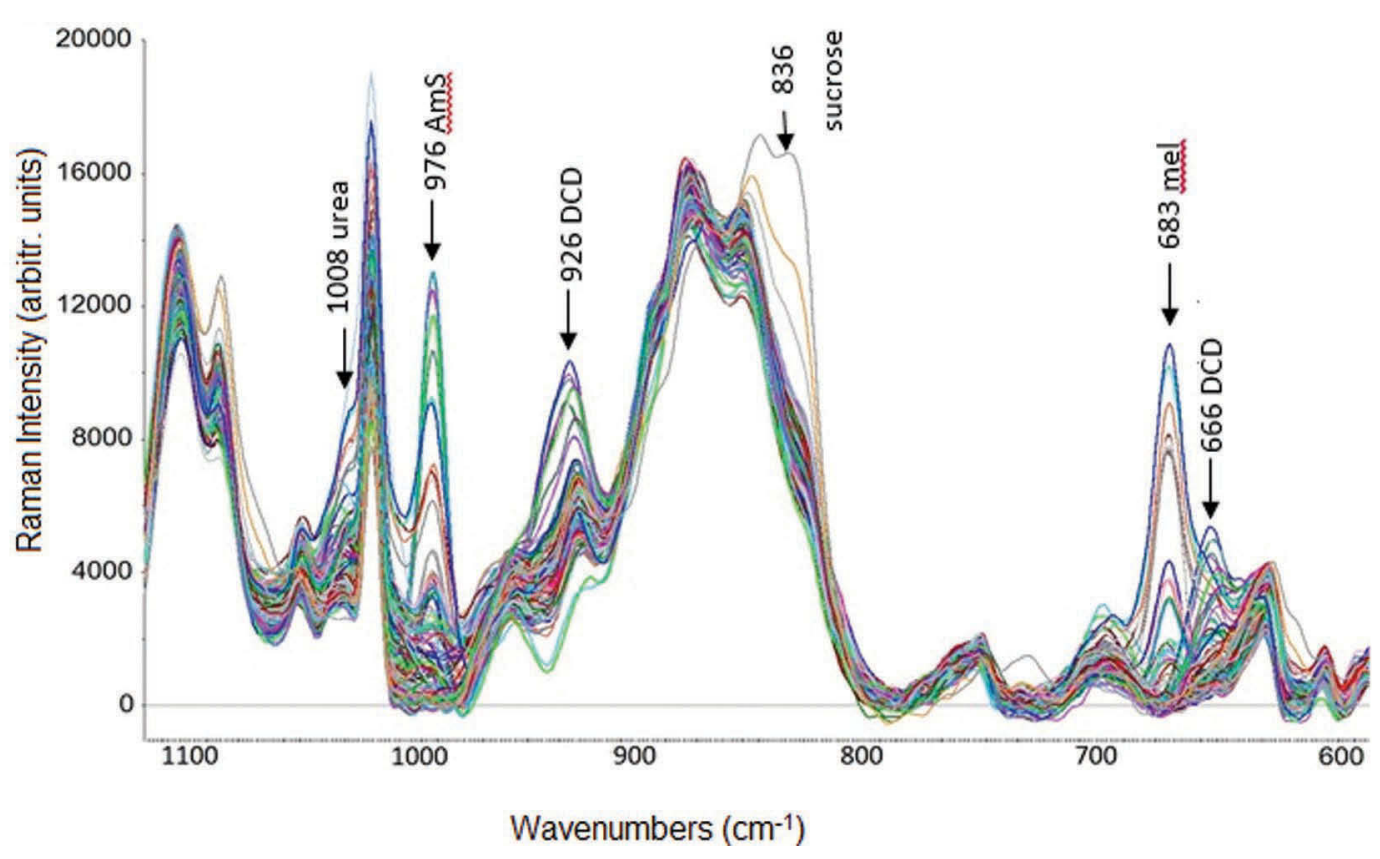

Figure 4. Overlaid spectra (124) used for partial least squares (PLS) analysis and partial least squares with discriminant analysis (PLS-DA) after baseline correction and smoothing using the Savitsky-Golay method with 9 points and a second-order polynomial. Color version available online.

are plotted in Figure 6, and the regression statistics are given in Table 3 . The RPD for the sucrose PLS calibration model was excellent $(>8.1)$, allowing it to be used for any application in the concentration range from 0 to $40,000 \mathrm{mg} / \mathrm{L}$ (or 0 to $4 \%$ ). For melamine, the RPD was very good (6.5 to 8.0) and suitable for process control, whereas that for DCD was good (5.6 to 6.4), making it suitable for quality control. The fair RPD values for AmS and urea indicate that these PLS calibration models would be suitable for screening only.

The LOD intervals for each compound and the parameters used to determine them from the PLS calibrations are given in Table 4. The LOD values determined from the univariate calibrations are included in the table for comparison. The $\mathrm{LOD}_{\min }$ and $\mathrm{LOQ}_{\min }$ values determined for the PLS calibration models were less than half those determined from the univariate measurements. The exceptions were for DCD and sucrose, where the univariate LOD value was closer to the $\mathrm{LOD}_{\min }$ for the PLS calibration; this may be due to better SNR observed for these compounds. For DCD, the univariate LOD was determined using the strong and isolated peak at $2,198 \mathrm{~cm}^{-1}$ in a different spectral region than used for the PLS calibration; for sucrose, a much larger concentration range was used. The $\mathrm{LOD}_{\min }$ sets the threshold level below which the analyte can be declared absent and should be more reliable than the univariate LOD values, considering the robustness of the PLS method in discounting instrument noise and inaccuracies from peak overlap. The $100 \mathrm{mg} / \mathrm{L} \mathrm{LOD}_{\text {min }}$

Table 2. Concentration of each contaminant determined from spectra of the cream fraction, using calibration plots of peak height versus concentration

\begin{tabular}{llcc}
\hline & & \multicolumn{2}{c}{ Amount remaining in cream fraction } \\
\cline { 3 - 4 } Adulterant & $\begin{array}{l}\text { Concentration } \\
\text { added }(\mathrm{mg} / \mathrm{L})\end{array}$ & Concentration $(\mathrm{mg} / \mathrm{L})$ & $\%$ \\
\hline Melamine $\left(683 \mathrm{~cm}^{-1}\right)$ & 3,000 & 783 & 26 \\
$\operatorname{AmS}^{1}\left(976 \mathrm{~cm}^{-1}\right)$ & 3,000 & 251 & 8.4 \\
$\operatorname{DCD}^{2}\left(2,198 \mathrm{~cm}^{-1}\right)$ & 5,000 & 1,019 & 20 \\
$\operatorname{DCD}^{-1}\left(924 \mathrm{~cm}^{-1}\right)$ & 3,000 & 641 & 21 \\
Urea $\left(1,008 \mathrm{~cm}^{-1}\right)$ & 3,000 & 424 & 14 \\
\hline
\end{tabular}

${ }^{1}$ Ammonium sulfate.

${ }^{2}$ Dicyandiamide. 
obtained here for DCD is very close to the $100 \mathrm{mg} / \mathrm{L}$ value obtained in a recent study using SERS on diluted milk (Lin et al., 2015).

The $\mathrm{LOD}_{\max }$ values were 2 to 3 times the $\mathrm{LOD}_{\min }$ values and set the upper threshold level above which the analyte can be verified as present; $\mathrm{LOD}_{\max }$ ranged from 310 to $450 \mathrm{mg} / \mathrm{L}$ for the $4 \mathrm{~N}$-rich compounds and was $2.5 \%$ for sucrose.

\section{PLS-DA for Screening of Adulterated Milk Samples}

A PC1 versus PC2 plot of an exploratory PCA analysis of the entire data set of 120 spectra showed that the samples of the adulterated milk solutions were dispersed along the PC1 axis according to concentra- tion of components. However, many of the samples below $500 \mathrm{mg} / \mathrm{L}$ were grouped together with more than one-third of the pure milk samples. Therefore, PLS-DA was needed to obtain better discrimination between the pure and adulterated milk samples. A PLS-DA calibration model was calculated using the training set of 80 samples, of which 24 were pure milk spectra and 56 were samples adulterated with one or mixtures of melamine, DCD, AmS, and urea. Ten PC were necessary and explained $80 \%$ of the data variance. The model was used to predict the separate validation set consisting of 11 pure milk samples and 33 adulterated samples, 4 of which were sucrose milk solutions at concentrations ranging from 2,500 to $20,000 \mathrm{mg} / \mathrm{L}$ (Figure 7). The predicted values for the pure milk samples were centered
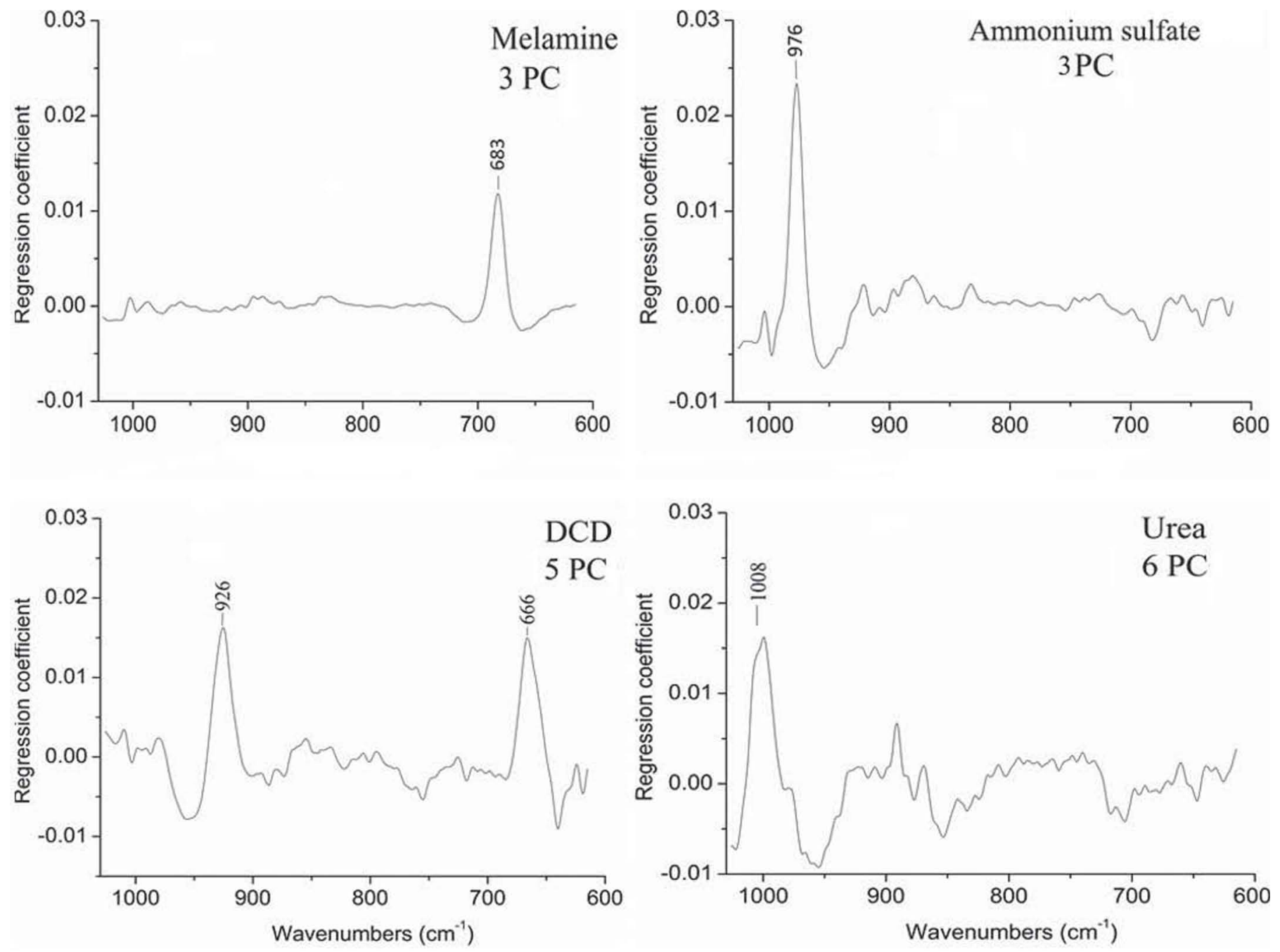

Figure 5. Regression coefficients for melamine, ammonium sulfate, dicyandiamide (DCD), and urea, showing the bands corresponding to the vectors used by the models to calculate the concentrations. The number of principal components (PC) used in each partial least squares (PLS) calibration are also indicated. 
Table 3. Root mean squared error of cross validation (RMSECV), $\mathrm{R}^{2}$, standard error of prediction (SEP) and ratio of SEP to SD (RPD) for partial least squares 1 calibrations of melamine, urea, dicyandiamide (DCD), and ammonium sulfate (AmS) between 0 and 1,000 mg/L and sucrose between 0 and $40,000 \mathrm{mg} / \mathrm{L}$

\begin{tabular}{|c|c|c|c|c|c|}
\hline Item & Melamine & $\mathrm{AmS}$ & DCD & Urea & Sucrose \\
\hline $\mathrm{R}^{2}$ (internal cross validation) & 0.97 & 0.93 & 0.97 & 0.92 & 0.99 \\
\hline $\mathrm{R}^{2}$ (external validation set) & 0.98 & 0.94 & 0.97 & 0.93 & - \\
\hline RPD & 7.0 & 4.4 & 5.9 & 4.0 & 13.2 \\
\hline Applicability & Process control & Screening only & Quality control & Screening only & Any application \\
\hline
\end{tabular}

${ }^{1}$ For sucrose, the SEP was determined from a leave-one-out full internal cross validation.
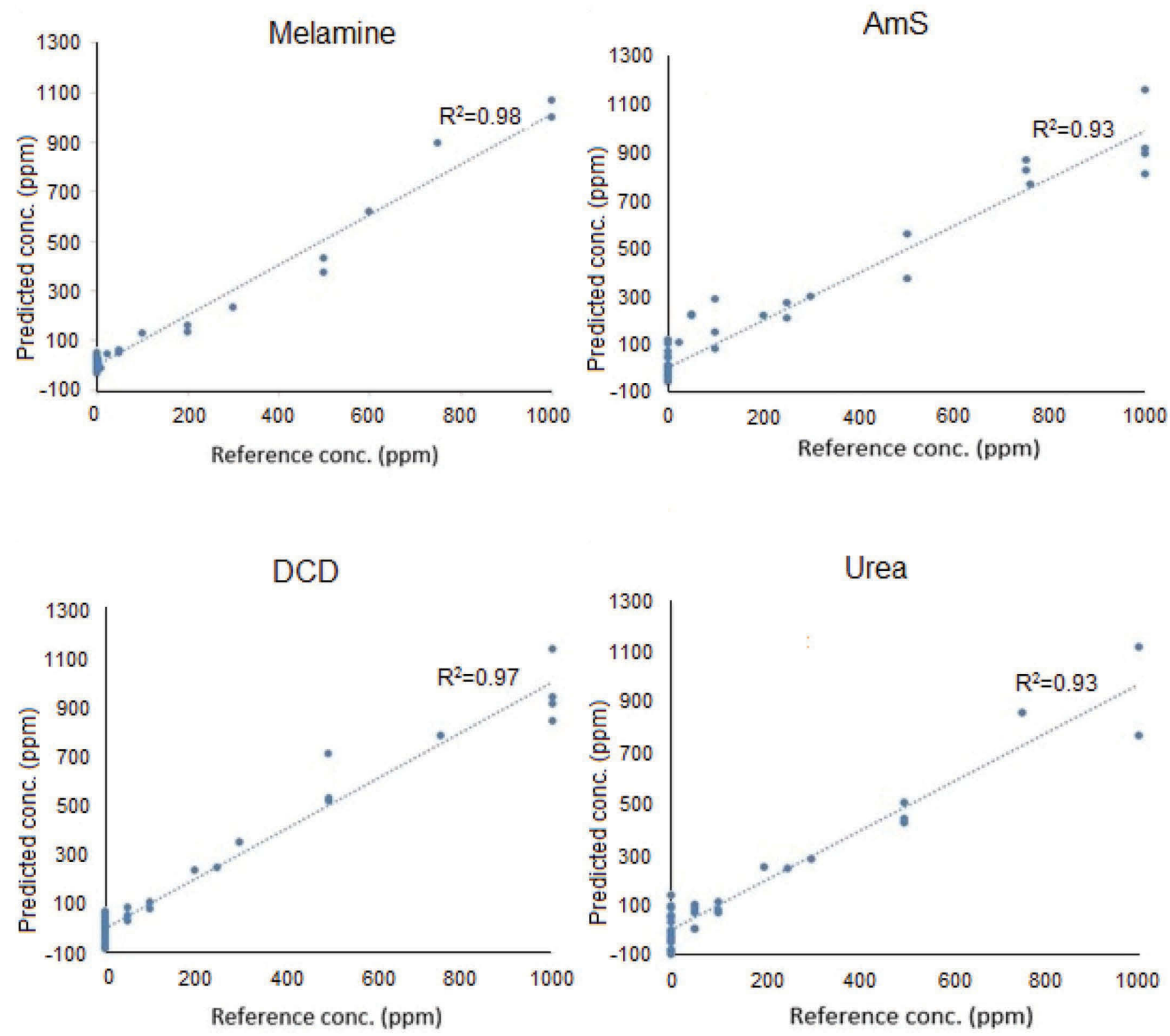

Figure 6. Predicted versus reference plots for partial least squares (PLS) prediction of the separate validation set for melamine, ammonium sulfate $(\mathrm{AmS})$, dicyandiamide (DCD), and urea. Color version available online. 
Table 4. Limit of detection (LOD) and limit of quantification (LOQ) intervals (minimum and maximum) for melamine, ammonium sulfate (AmS), dicyandiamide (DCD), urea, and sucrose partial least squares calibrations

\begin{tabular}{lcccrr}
\hline Item $^{1}$ & $\begin{array}{c}\text { Melamine } \\
(\mathrm{mg} / \mathrm{L})\end{array}$ & $\begin{array}{c}\mathrm{AmS} \\
(\mathrm{mg} / \mathrm{L})\end{array}$ & $\begin{array}{c}\text { DCD } \\
(\mathrm{mg} / \mathrm{L})\end{array}$ & $\begin{array}{c}\text { Urea } \\
(\mathrm{mg} / \mathrm{L})\end{array}$ & $\begin{array}{c}\text { Sucrose } \\
(\mathrm{mg} / \mathrm{L})\end{array}$ \\
\hline LOD $_{\min }$ & 100 & 150 & 140 & 120 & 7,205 \\
LOD $_{\max }$ & 260 & 350 & 600 & 490 & 25,245 \\
LOD univariate $_{\text {LOQ }}$ & 180 & 230 & 130 & 280 & 7,290 \\
LOQ $_{\max }$ & 310 & 450 & 440 & 350 & 21,834 \\
LOQ univariate & 800 & 1,070 & 1,810 & 1,490 & 76,501 \\
\hline
\end{tabular}

${ }^{1} \mathrm{LOD}$ and LOQ determined from the univariate analysis are included for comparison.

around -1 , and the adulterated samples around +1 , except for the 2 high concentrations of sucrose. The threshold limit was set at 0 , as it halfway between these 2 , excluding those of sucrose, which was not included in the training set. High values were predicted for the 15,000 and $20,000 \mathrm{mg} / \mathrm{L}$ sucrose samples, as they were very different from the calibration set.

Figures of merit such as false positive rate, false negative rate, and efficiency were calculated to evaluate the effectiveness of the PLS-DA calibration model for screening milk. The false positive, false negative, and efficiency rates express the trueness in qualitative analysis, and the selectivity rate measures the selectivity of the method (Botelho et al., 2015). The PLS-DA model correctly predicted $100 \%$ of the adulterated samples, with a selectivity rate of $100 \%$, and false negative rate of $0 \%$. The incorrect prediction of 2 false positives resulted in a false positive rate of $15.4 \%$ and an efficiency rate of $84.6 \%$. All of the sucrose samples were correctly identified as adulterated, even though no sucrose solutions were included in the training set.

\section{CONCLUSIONS}

The results show that Raman spectroscopy with PLS and PLS-DA is feasible for fast, reliable, and sen-

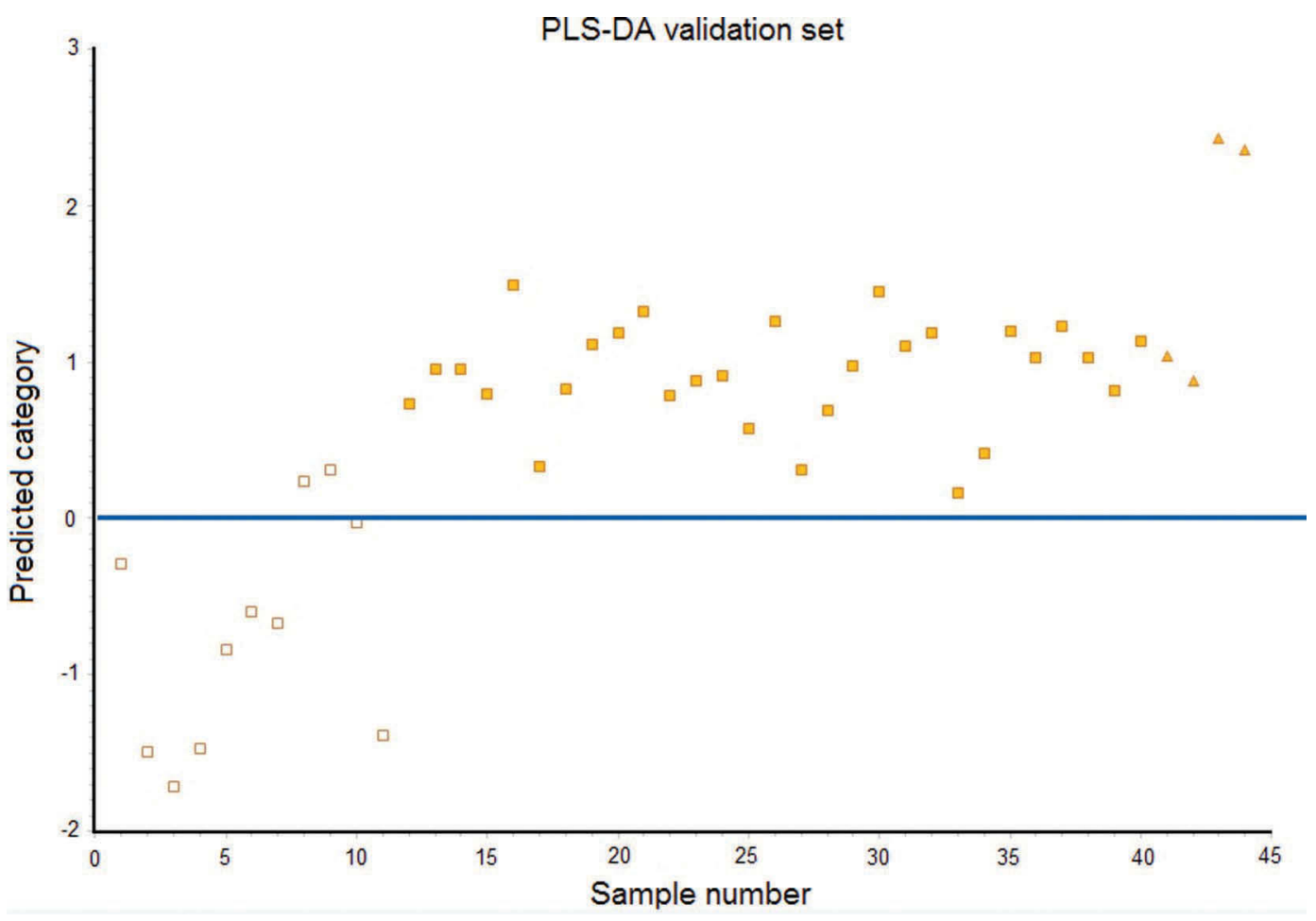

Figure 7. Partial least squares with discriminant analysis (PLS-DA) prediction of the validation set consisting of 11 pure milk samples ( $\square$ ), 29 milk samples adulterated with one or more of melamine, urea, dicyandiamide, and ammonium sulfate (ם) and 4 samples adulterated with sucrose $(\boldsymbol{\Lambda})$. Samples with a value greater than zero are predicted to be adulterated and samples with a value less than zero are considered pure. Color version available online. 
sitive screening of milk for adulteration of milk with the N-rich compounds melamine, ammonium sulfate, urea, and DCD, even at low levels of 50 to $1,000 \mathrm{mg} / \mathrm{L}$ $(0.05-0.1 \%, \mathrm{wt} / \mathrm{wt})$. Spectra were recorded from dried drops of milk with no need for sample preparation or addition of chemicals, making this a greener technique. Reliable and consistent sample presentation enabled an excellent reproducibility of $7 \%$ RSD. Limits of detection obtained from the PLS calibrations were determined as interval-specific for the sample concentration range, with an average $\mathrm{LOD}_{\min }$ of $128 \mathrm{mg} / \mathrm{L}$ for the N-rich compounds and $7,250 \mathrm{mg} / \mathrm{L}(0.7 \%$, wt/wt) for sucrose and $\mathrm{LOD}_{\max } 2$ to 3 times these values. Simultaneous analysis of the 4 small N-rich molecules in milk was possible at these low levels due to the high selectivity of Raman spectroscopy and the use of multivariate analysis. With the increasing availability of competitively priced portable mini-Raman systems, the technique shows promise for deployment on-site for rapid and reliable detection of adulterated milk and is easily incorporated into microfluidics.

\section{ACKNOWLEDGMENTS}

This work was supported by the Primary Growth Partnership (PGP) scheme sponsored by Fonterra Cooperative Group (New Zealand) and the New Zealand Government (Wellington, New Zealand).

\section{REFERENCES}

Abbas, O., L. Bernard, P. Dardenne, and V. Baeten. 2013. Detection of melamine and cyanuric acid in feed ingredients by near infrared spectroscopy and chemometrics. J. Near Infrared Spectrosc. $21: 183-194$.

Abernethy, G., and K. Higgs. 2013. Rapid detection of economic adulterants in fresh milk by liquid chromatography-tandem mass spectrometry. J. Chromatogr. A 1288:10-20.

Allegrini, F., and A. C. Olivieri. 2014. IUPAC-consistent approach to the limit of detection in partial least-squares calibration. Anal. Chem. 86:7858-7866.

Ashok, P. C., G. P. Singh, K. M. Tan, and K. Dholakia. 2010. Fiber probe based microfluidic Raman spectroscopy. Opt. Express 18:7642-7649.

Balabin, R. M., and S. V. Smirnov. 2011. Melamine detection by midand near-infrared (MIR/NIR) spectroscopy: A quick and sensitive method for dairy products analysis including liquid milk, infant formula and milk powder. Talanta 85:562-568.

Borin, A., M. F. Ferrao, C. Mello, D. A. Maretto, and R. J. Poppi. 2006. Least-squares support vector machines and near infrared spectroscopy for quantification of common adulterants in powdered milk. Anal. Chim. Acta 579:25-32.

Botelho, B. G., N. Reis, L. S. Oliveira, and M. M. Sena. 2015. Development and analytical validation of a screening method for simultaneous detection of five adulterants in raw milk using mid-infrared spectroscopy and PLS-DA. Food Chem. 181:31-37.

Cattaneo, T., and S. Holroyd. 2013. Review: The use of near infrared spectroscopy for determination of adulteration and contamination in milk and milk powder: Updating knowledge. J. Near Infrared Spectrosc. 21:341-349.
Chan, E. Y., S. Griffiths, and C. Chan. 2008. Public-health risks of melamine in milk products. Lancet 372:1444-1445.

Chao, K., J. Qin, M. S. Kim, Y. Peng, D. Chan, and Y. C. Cheng. 2013. Raman spectroscopy and imaging to detect contaminants for food safety applications. Pages 87210 S1-87210S10 in SPIE Proc. 8721: Sensing for Agriculture and Food Quality and Safety V SPIE, Washington, DC

Chávez-Servín, J. L., A. I. Castellote, and M. C. López-Sabater. 2004 Analysis of mono- and disaccharides in milk-based formulae by high-performance liquid chromatography with refractive index detection. J. Chromatogr. A 1043:211-215.

Cheng, Y., and Y. Dong. 2011. Screening melamine contaminant in eggs with portable surface-enhanced Raman spectroscopy based on gold nanosubstrate. Food Contr. 22:685-689.

Cheng, Y., Y. Dong, J. Wu, X. Yang, H. Bai, H. Y. Zheng, D. Ren, Y. Zou, and M. Li. 2010. Screening melamine adulterant in milk powder with laser Raman spectrometry. J. Food Compos. Anal 23:199-202.

Cheng, Y., J. Qin, J. Lim, D. E. Chan, M. S. Kim, and K. Chao. 2012. An investigation of FT-Raman spectroscopy for quantification of additives to milk. Pages 83690W1-83690W9 in Proc. SPIE Vol. 8369. Sensing for Agriculture and Food Quality and Safety IV Baltimore, MD. SPIE, Washington, DC.

Chrimes, A. F., K. Khoshmanesh, P. R. Stoddart, A. Mitchell, and K. Kalantar-Zadeh. 2013. Microfluidics and Raman microscopy: Current applications and future challenges. Chem. Soc. Rev. 42:58805906.

Codex Alimentarius. 2010. Codex Committee on Contaminants in Foods: Proposed draft maximum levels for melamine in food and feed. Proc. Joint FAO/WHO Food Standards Programme. Codex Alimentarius Commission, Izmir, Turkey.

Delhaye, M., J. Barbillat, J. Aubard, M. Bridoux, and E. da Silva. 1996. Instrumentation. Chapter 3 in Raman Microscopy: Developments and Applications. J. C. G. Turrell and J. Corset, ed. Academic Press Ltd., London, UK.

Desmarchelier, A., M. Guillamon Cuadra, T. Delatour, and P. Mottier. 2009. Simultaneous quantitative determination of melamine and cyanuric acid in cow's milk and milk-based infant formula by liquid chromatography-electrospray ionization tandem mass spectrometry. J. Agric. Food Chem. 57:7186-7193.

Domingo, E., A. A. Tirelli, C. A. Nunes, M. C. Guerreiro, and S. M. Pinto. 2014. Melamine detection in milk using vibrational spectroscopy and chemometrics analysis: A review. Food Res. Int 60:131-139.

Food Safety and Standard Authority of India. 2012. Milk and milk products. Pages 8-12 in Manual of Methods of Analysis of Food. Food Safety and Standard Authority, New Delhi, India.

Gem, H. 1857. The adulteration of milk. Lancet Feb. 7:156

Guicheteau, J., S. Christesen, D. Emge, P. Wilcox, and A. W. Fountain. 2011. Assessing metal nanofabricated substrates for surfaceenhanced Raman scattering (SERS) activity and Reproducibility. Appl. Spectrosc. 65:144-151.

ICH (International Conference on Harmonisation). 1996. Guidance for Industry, Q2B Validation of Analytical Procedures: Methodology. U.S. Department of Health and Human Services, Food and Drug Administration, Center for Drug Evaluation and Research (CDER), Center for Biologics Evaluation and Research (CBER), Silver Spring, MD.

Jawaid, S., F. N. Talpur, S. T. Sherazi, S. M. Nizamani, and A. A. Khaskheli. 2013. Rapid detection of melamine adulteration in dairy milk by SB-ATR-Fourier transform infrared spectroscopy. Food Chem. 141:3066-3071.

Johansson, J., A. Sparen, O. Svensson, S. Folestad, and M. Claybourn. 2007. Quantitative transmission Raman spectroscopy of pharmaceutical tablets and capsules. Appl. Spectrosc. 61:1211-1218.

Kettle, S. F. A., U. A. Jayassoriya, and L. J. Norrby. 1984. Reinterpretation of the internal mode vibrational spectra of ammonium sulfate (Phase I). J. Phys. Chem. 88:5971-5975.

Keuleers, R., H. O. Desseyn, B. Rousseau, and C. Van Alsenoy. 1999. Vibrational analysis of urea. J. Phys. Chem. A 103:4621-4630. 
Koglin, E., J. Kip, and R. J. Meier. 1996. Adsorption and displacement of melamine at the $\mathrm{Ag} /$ electrolyte interface probed by surface-enhanced Raman microprobe spectroscopy. J. Phys. Chem. 100:5078-5089.

Lin, X., W.-L.-J. Hasi, X.-T. Lou, S. Han, D.-Y. Lin, and Z.-W. Lu. 2015. Direct and quantitative detection of dicyandiamide (DCD) in milk using surface-enhanced Raman spectroscopy. Anal. Methods 7:3869-3875.

Mauer, L. J., A. Chernyshova, A. Hiatt, A. Deering, and R. Davis. 2009. Melamine detection in infant formula powder using near- and mid-infrared spectroscopy. J. Agric. Food Chem. 57:3974-3980.

McCreery, R. L. 2000. Raman Spectroscopy for Chemical Analysis. 3rd ed. Wiley, New York.

Moore, J. C., J. W. DeVries, M. Lipp, J. C. Griffiths, and D. C. Abernethy. 2010. Total protein methods and their potential utility to reduce the risk of food protein adulteration. Compr. Rev. Food Sci. Food Safety 9:330-357.

Moore, J. C., J. Spink, and M. Lipp. 2012. Development and application of a database of food ingredient fraud and economically motivated adulteration from 1980 to 2010. J. Food Sci. 77:R118-R126.

Moskovits, M. 2013. Persistent misconceptions regarding SERS. Phys. Chem. Chem. Phys. 15:5301-5311.

New York Times. 1890. Milk adulteration. Accessed Jan. 16, 2016. http://query.nytimes.com/mem/archive-free/pdf?res=9C03E2D7 133BE533A25750C1A96E9C94619ED7CF.

Norrod, K. L., L. M. Sudnik, D. Rousell, and K. L. Rowlen. 1997. Quantitative comparison of five SERS substrates: Sensitivity and limit of detection. Appl. Spectrosc. 51:994-1001.

Okazaki, S., M. Hiramatsu, K. Gonmori, O. Suzuki, and A. T. Tu. 2009. Rapid nondestructive screening for melamine in dried milk by Raman spectroscopy. Forensic Toxicol. 27:94-97.

Qin, J., K. Chao, Y.-C. Cheng, and M. S. Kim. 2013a. Authentication of milk powder using Raman scattering spectroscopy and imaging. Paper 131591839, pages 1-9, in Proc. 2013 ASABE Annu. Int. Mtg., Kansas City, MO. ASABE, St. Joseph, MI.

Qin, J., K. Chao, and M. S. Kim. 2010. Raman chemical imaging system for food safety and quality inspection. Am. Soc. Agric. Biol. Eng. 53:1873-1882.
Qin, J., K. Chao, and M. S. Kim. 2010. Raman chemical imaging system for food safety and quality inspection. Trans. ASABE 53:1873-1882.

Qin, J., K. Chao, and M. S. Kim, 2012. Detecting multiple adulterants in dry milk using Raman chemical imaging. Pages 83690H183690H12 in Proc. SPIE 8369: Sensing for Agriculture and Food Quality and Safety IV 8369. SPIE, Washington, DC.

Qin, J., K. Chao, and M. S. Kim. 2013b. Simultaneous detection of multiple adulterants in dry milk using macro-scale Raman chemical imaging. Food Chem. 138:998-1007.

Rajapandiyan, P., W.-L. Tang, and J. Yang. 2015. Rapid detection of melamine in milk liquid and powder by surface-enhanced Raman scattering substrate array. Food Contr. 56:155-160.

Santos, P. M., E. R. Pereira-Filho, and L. E. Rodriguez-Saona. 2013. Rapid detection and quantification of milk adulteration using infrared microspectroscopy and chemometrics analysis. Food Chem. 138:19-24.

Srilakshmi, C., E. Widjaja, B. G. Anderson, and M. Garland. 2007. Fourier transform Raman spectral measurements of powdered quaternary mixtures of organic compounds Exceptional pure component spectral reconstruction using band-target entropy minimization (BTEM). Talanta 72:847-853.

WHO. 2009. Toxicological and health aspects of melamine and cyanuric acid. Report of a WHO expert meeting, in collaboration with the Food and Agriculture Organization of the United Nations (Rome, Italy) and supported by Health Canada (Ottawa, ON, Canada)

Williams, P. 2008. The RPD: What is it, where did it come from, and what does it mean? Pages 48-49 in Proc. 13th Australian Near Infrared Spectroscopy Conf., Hamilton, Victoria, Australia. IM Publications LLP, West Sussex, UK.

Zhao, Y., W. Luo, P. Kanda, H. Cheng, Y. Chen, S. Wang, and S. Huan. 2013. Silver deposited polystyrene (PS) microspheres for surface-enhanced Raman spectroscopic-encoding and rapid labelfree detection of melamine in milk powder. Talanta 113:7-13. 


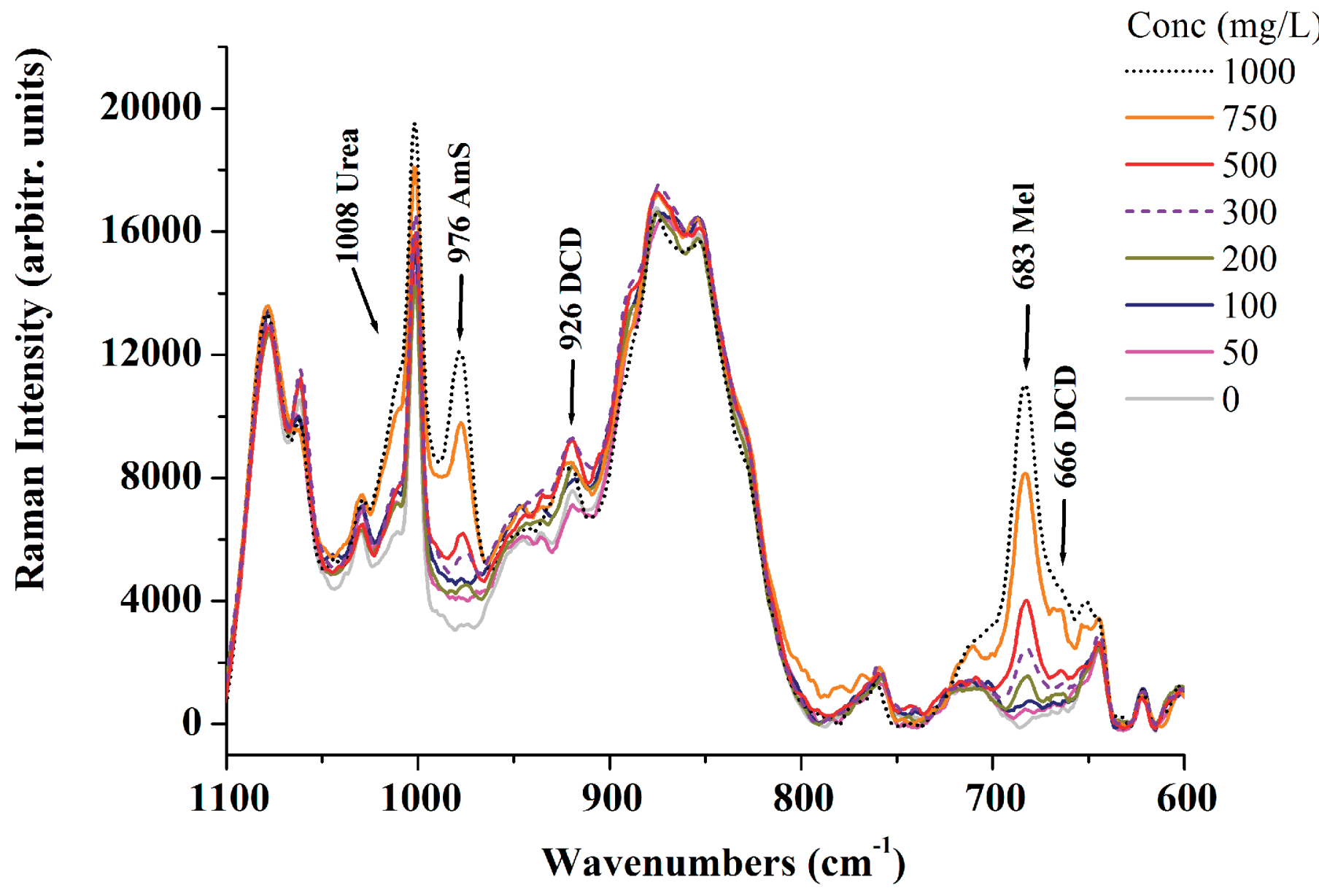

Figure A1. The spectra of pure milk and milk spiked with equal mixtures of melamine, ammonium sulfate (AmS), dicyandiamide (DCD), and urea at concentrations of $0,50,100,200,300,500,750$, and 1,000 $\mathrm{mg} / \mathrm{L}$. Color version available online. 

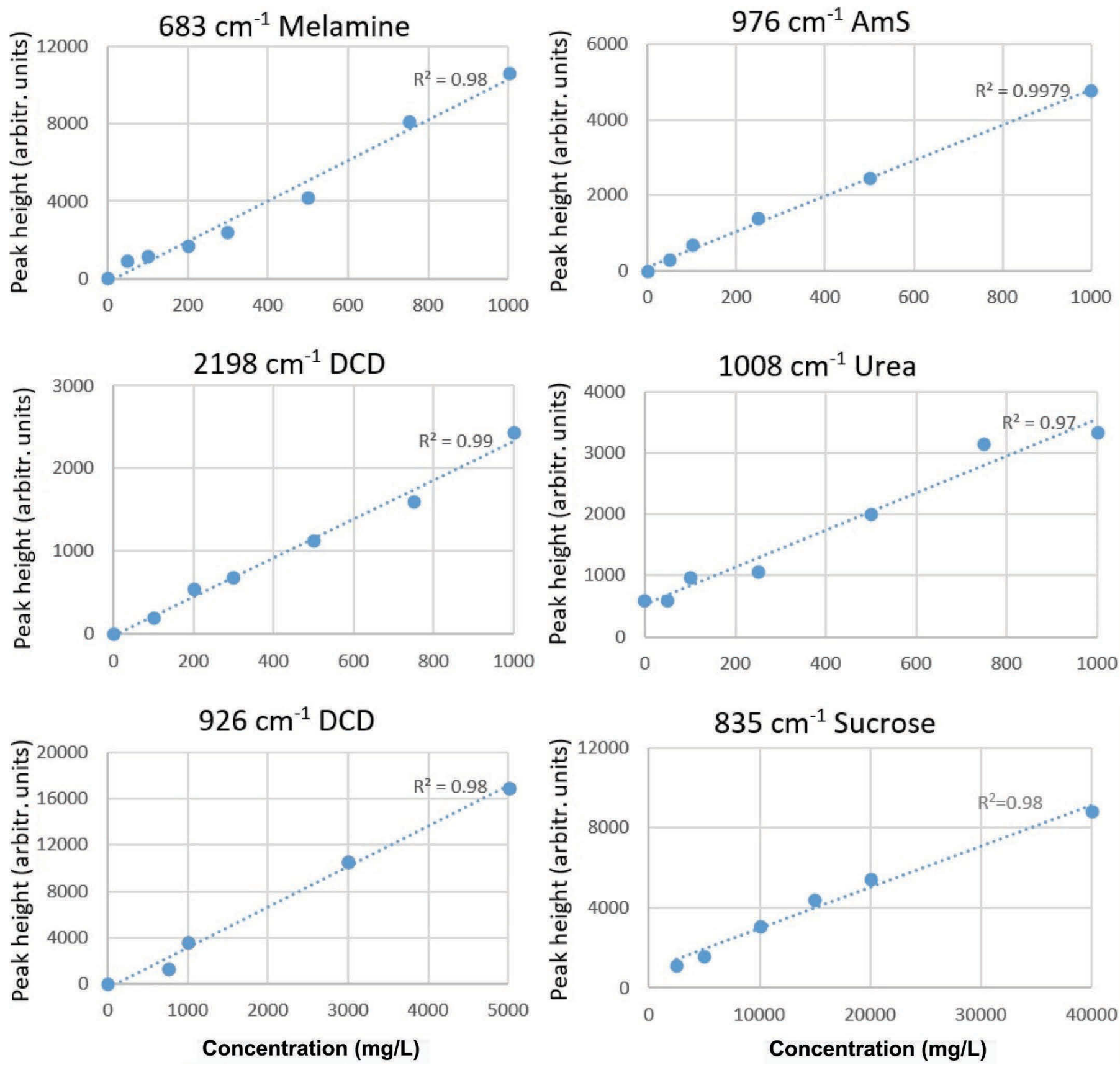

Figure A2. Univariate calibration plots of peak height versus concentration (mg/L) for melamine, ammonium sulfate (AmS), dicyandiamide (DCD), urea, and sucrose. Color version available online. 


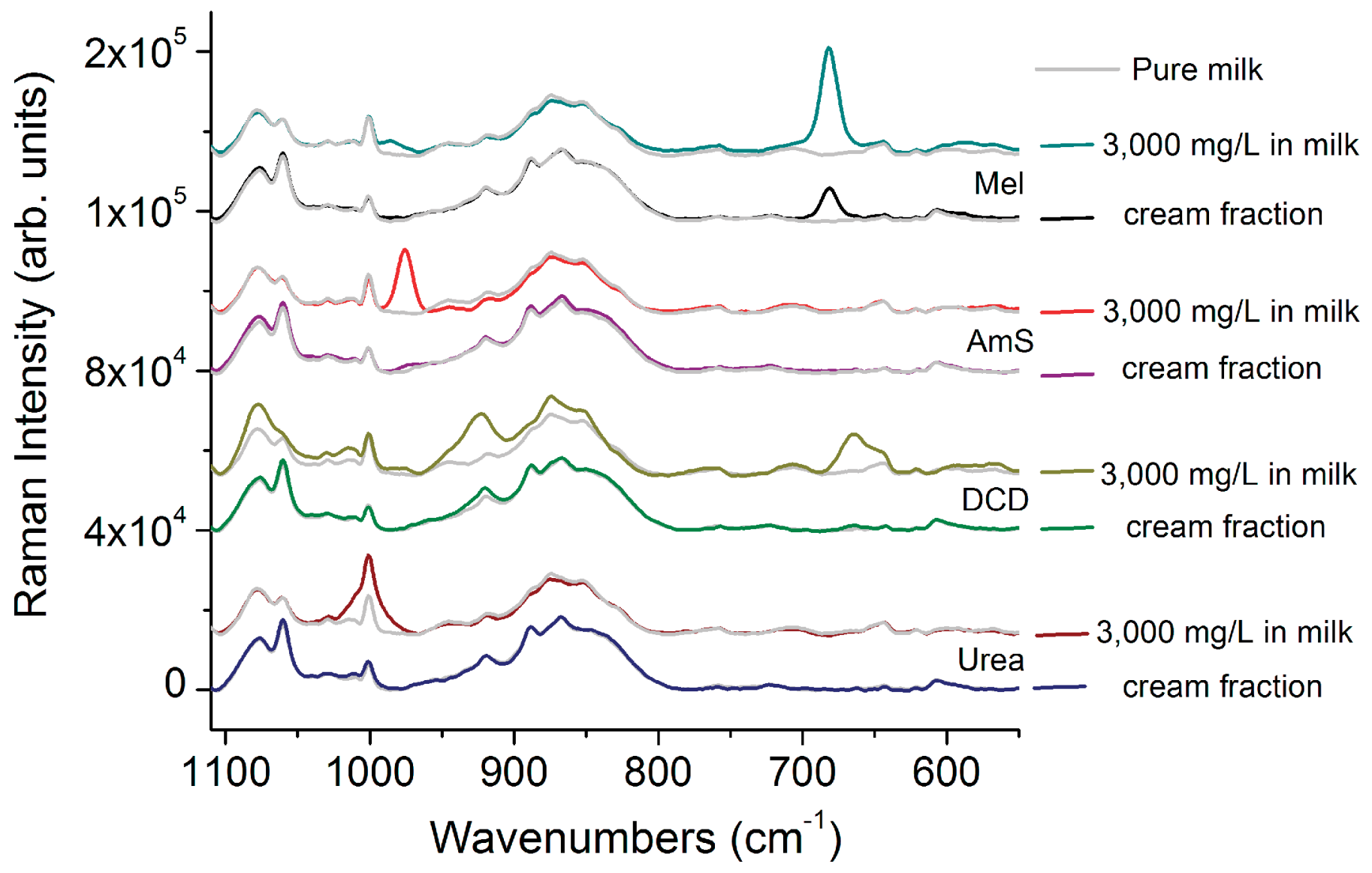

Figure A3. Spectra of 3,000 mg/L solutions of melamine (Mel), ammonium sulfate (AmS), and urea and 5,000 mg/L solutions of dicyandiamide (DCD) in milk before centrifuging, and the cream fractions from each after centrifuging at 8,050 $\times g$ for 15 min. Overlaid with each spectrum is a spectrum of pure milk (light gray) for comparison. Color version available online. 